\title{
Real-Time Stability Assessment based on Synchrophasors
}

Jóhannsson, Hjörtur; Garcia-Valle, Rodrigo; Weckesser, Johannes Tilman Gabriel; Nielsen, Arne Hejde; Østergaard, Jacob

Published in:

IEEE PES Trondheim PowerTech

Publication date:

2011

Link back to DTU Orbit

Citation (APA):

Jóhannsson, H., Garcia-Valle, R., Weckesser, J. T. G., Nielsen, A. H., \& Østergaard, J. (2011). Real-Time Stability Assessment based on Synchrophasors. In IEEE PES Trondheim PowerTech IEEE.

http://ewh.ieee.org/conf/powertech/2011/

\section{General rights}

Copyright and moral rights for the publications made accessible in the public portal are retained by the authors and/or other copyright owners and it is a condition of accessing publications that users recognise and abide by the legal requirements associated with these rights.

- Users may download and print one copy of any publication from the public portal for the purpose of private study or research.

- You may not further distribute the material or use it for any profit-making activity or commercial gain

- You may freely distribute the URL identifying the publication in the public portal

If you believe that this document breaches copyright please contact us providing details, and we will remove access to the work immediately and investigate your claim 


\title{
Real-Time Stability Assessment based on Synchrophasors
}

\author{
Hjörtur Jóhannsson, Rodrigo Garcia-Valle Member, IEEE, Johannes Tilman Gabriel Weckesser, \\ Arne Hejde Nielsen Senior Member, IEEE, Jacob Østergaard Senior Member, IEEE
}

\begin{abstract}
In this paper, an overview is provided of a new method that in real-time provides an early warning for an emerging blackout that are characterized by a slowly increasing angular separation between sub-groups of system generators. Such angular separation between subgroups of generators can eventually cause in very sharp decline in system voltages at intermediate locations between the two groups as the angular separation approaches $180^{\circ}$. In order to receive an early warning for the occurrence of such type of blackouts, the boundaries of the system generators aperiodic small-signal stability are suggested to be monitored. For that purpose, method for real-time assessment of aperiodic small-signal rotor angle stability is presented. The approach is based on an element-wise assessment of individual synchronous machines where the aim is to determine the maximum steady state power that each synchronous generator can inject into the system. The limits for maximum injectable power represent the boundary for aperiodic small signal stability.

The concept of the proposed method is tested on two different systems. The results show that the method is capable of accurately detecting when a given machine crosses the stability boundary. The method can as well provide in real-time a margin to the machines stability boundary, which can be used as an early warning for an impending system stability problem.
\end{abstract}

Index Terms-Phasor measurement units, Power system stability, Power system security

\section{INTRODUCTION}

The stable and secure operation of electric power systems are topics of fundamental importance to the modern society and will continue to be so in the future. The global challenges for electric power systems in the coming decades are great and will focus on the establishment of energy supply that has a minimum dependency on fossil fuels. The challenges involve the development of future power systems that can supply an adequate and stable energy service at competitive prices and with minimal environmental impact. For meeting those challenges, it is expected that the share of power production capacity which is based on non-controllable renewable energy sources will drastically increase in the coming decades.

In a system, where significant part of the power production subject to prevailing weather conditions, increased fluctuations of the system operating point will be observed. This makes the planning of stable and secure operation a challenging task and in fact, might cause that planning could no longer be done few hours ahead. This is due to the uncertainty in the estimation of the system production patterns for few hours ahead and leads to that conventional means for operational planning will not

The authors are with the Centre of Electric Technology, Department of Electrical Engineering, the Technical University of Denmark be adequate to ensure system stability and security. Therefore, methods for real-time assessment of security margins and the closeness to stability boundaries are needed.

Over the many years of research and development, sophisticated computer tools have been developed for stability analysis. A general characteristic of these tools is that they are capable of determining whether a given condition is stable or unstable, but due to high computational burden, the stability assessment is carried out off-line. Consequently, these tools are not suitable for real-time assessment of the system stability.

The development of the phasor measurement technology [1], [2], [3] facilitated the research and development of monitoring and control applications that can utilize the wide area measurements of system voltages [4], [5].

Open literature gives a few methods, which aim at online security or stability assessment of a system condition observed by utilizing synchronized phasor measurement. In [6], [7], a method for early detection of impending voltage instability is presented. The method utilizes system snapshots for computing sensitivities for the purpose of assessing the system voltage stability. The method does not relay on a dynamic model to predict the system response, instead some basic assumptions and simplifications are applied to the system model resulting in reduced computational burden for the assessment.

In [8], an online dynamic security assessment tool presented that carries out an analysis for a snapshot of an observed system condition. The snapshot is used as a base case and the system security in respect to a wide variety of contingencies is investigated. The typical computation time for carrying out security assessment for an observed contingency is from 5 to 15 minutes. This time frame might become critical in systems having significant fluctuations of the system operating point.

In [9], a real-time approach for security assessment is presented, where a multidimensional security region is determined for a specified operational base case. The security region boundary is represented using its piecewise linear hyperplanes in a multi-dimensional space, consisting of system parameters that are critical for security analysis. The security regions are pre-calculated off-line in a time-consuming process where the security region is derived for one particular system configuration. A real-time assessment of the system security is obtained by monitoring the multidimensional operating point in realtime and comparing it against the pre-calculated boundaries. If the system is subjected to any topological change (e.g. tripped lines due to maintenance), the actual approach may introduce an uncertainty for the assessment of security margin, as it has been based on the non-changed topological structure. 
(a)

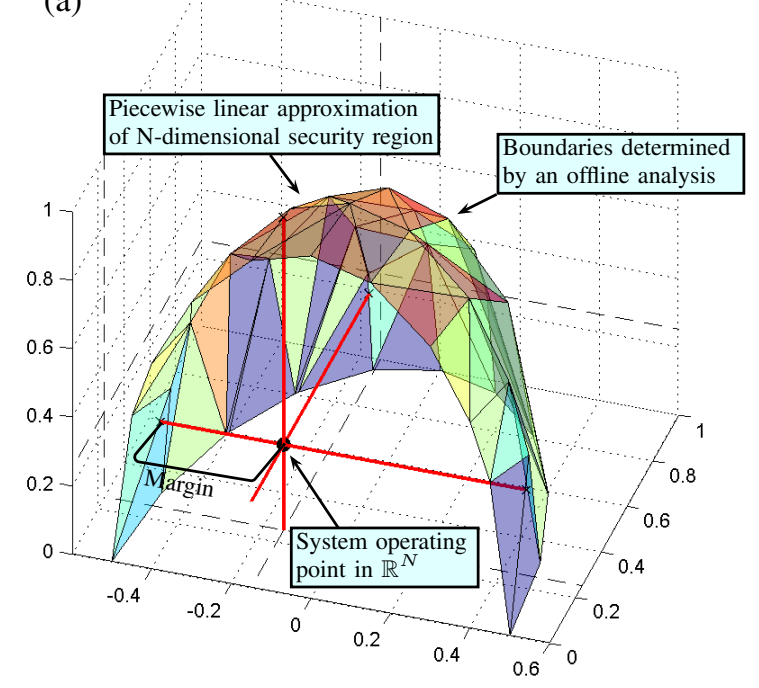

(b)

Element-wise Assessment

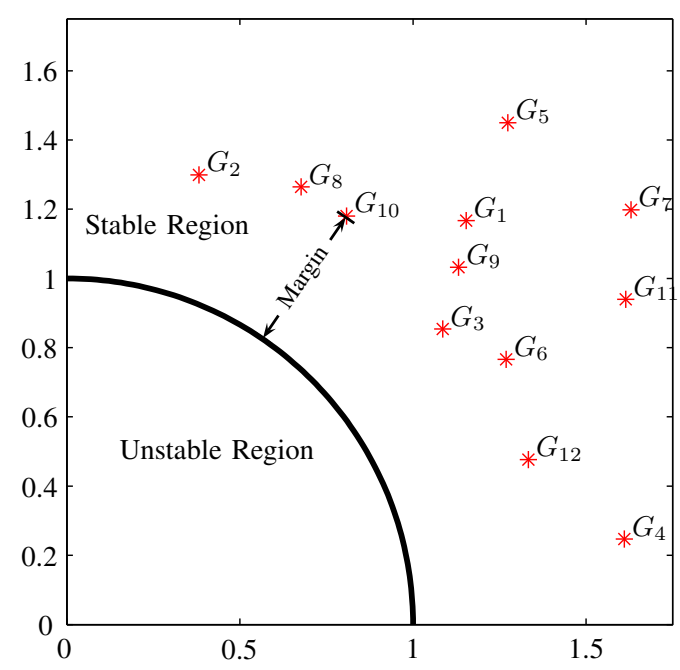

Fig. 1. Two different approaches for real-time assessment of stability or security boundaries. (a) Assessment of a multidimensional operating point (in $\mathbb{R}^{N}$ ) against its boundaries. The boundaries are determined by offline analysis, while the operating point is monitored in real-time. (b) Element-wise assessment of stability, where the one particular mechanism of instability is assessed explicitly for each relevant system element. An individual operating point is associated with each element (in this case generators $G_{i}$ ) is held against its stability boundary.

In this paper a distinctive approach for assessing realtime power system stability is presented. The main focus relies on element-wise assessment of individual mechanisms of stability, rather than carrying overall assessment of a single multi-dimensional operating point.

\section{MethoD}

Figure 1 illustrates the fundamental difference between the previous mentioned methods [8], [9] and the one presented within this paper. Figure 1.(a) illustrates a multidimensional boundary in $\mathbb{R}^{N}$ and its corresponding multi-dimensional operating point. The distance of the single operating point to the critical boundary represents the margin to an unstable (or insecure) operation. In [9] the boundaries are determined via off-line analysis, but the real-time assessment consists of monitoring the multi-dimensional operating point and its distance to the boundaries. The proposed method provides an overall assessment of the system stability (or security) by observing a single multi-dimensional operating point in $\mathbb{R}^{N}$.

Figure 1.(b) depicts the proposed concept for stability assessment applied in this paper. In this case, the stability of a given system element is evaluated in respect to a given mechanism of stability. This could be for example, the assessment of when generator $\left(G_{i}\right)$ reaches its limit of maximum injectable power in steady state condition. The limits for maximum injectable power represents the stability limit for aperiodic small signal stability for the machine of concern. This is approach is referred to as element-wise assessment. The concept is illustrated in this figure, where each operating point is associated to a generator $G_{i}$ and the point is held against a critical boundary for a given mechanism of stability (the boundary could be presented in $\mathbb{R}^{2}$ or even $\mathbb{R}$ ).

The element-wise approach provides only an assessment of one particular from of instability. Approach for full assessment of the system stability is outlined in Fig. 2. This approach is based on synchronized wide area observations that provide full system observability. This approach uses different

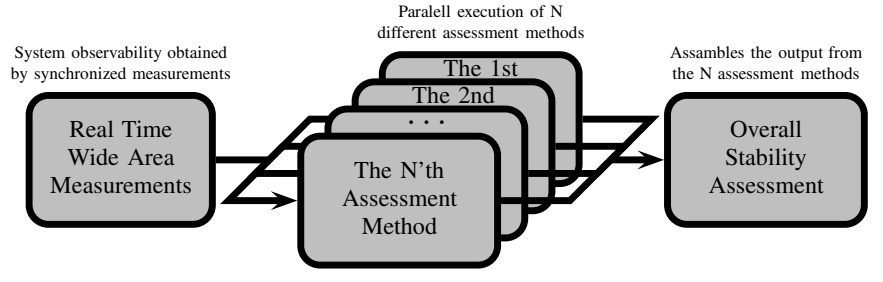

Fig. 2. Schematic overview over real time stability assessment process.

assessment methods, where each method is focused on one particular mechanism of stability. This might include a method that assesses the risk of cascading outages, a method that assesses the system voltage stability, a method that assesses the generators small signal rotor angle stability and so on. Each of these methods make use of a wide area snapshot of the system conditions, and therefore all methods are executed in parallel. The assessment output from each method is gathered and analyzed in order to obtain an overall stability assessment of the system conditions in real-time.

Several benefits will be obtained by splitting up the overall stability assessment problem when a real-time analysis of the system is carry out:

- The system description can be tailored to the stability mechanism that is being addressed. This enables model reduction where system dynamics that have limited or no effect on the addressed stability mechanism are neglected. Hence, computational efforts are considerably reduced.

- Since each assessment method is focussing on a particular mechanism of stability, it simple to identify where in the system a problem is emerging, and to which kind of stability problem is experienced.

- The parallel execution of several assessment methods improves the total execution time.

In the following, a method is presented that focusses on the assessment of one particular mechanism of stability; the capability of each generator to generate sufficient steady syn- 
chronizing torque such that a operation at stable equilibrium point can be maintained. The lack of sufficient steady state synchronizing torque causes aperiodic increase in rotor angle and eventually a loss of synchronism.

\section{A. Boundary of Aperiodic Small-Signal Rotor Angle Stability}

In the following, a method for determining boundaries of aperiodic small signal stability is presented. The method is based on that full observability of the system state is obtained by real-time measurements of the grid. The full network observability is used to establish a deterministic representation of the system conditions, where the following simplifying assumptions are made:

- The network is represented by an extended admittance matrix, where all power injections into the system enter the network in a node of constant steady state voltage magnitude

- Usually at the terminal of a generator or behind its saturated direct axis reactance $X_{d}^{s}$ depending on the type machine excitation control (automatic or manual excitation) and status of the machine overexcitation limiter (OEL).

- Results in additional nodes and branches to be included in the system admittance matrix for each manually excited machine

- The load is represented as impedances in the network and is included in the extended admittance matrix

- Longer term load dynamics not included.

- The method evaluates the instantiations operating conditions, therefore the instantaneous impedance as seen from the generators is represented.

By representing the power injections at nodes of constant steady state voltage magnitude results in a reduction of the degrees of freedom associated with the determination of the boundaries for aperiodic small signal stability for a given generator.

The boundary of an aperiodic small signal rotor angle stability depends on the maximum amount of power that a given generator can inject into a network in steady state.

In [10], an expression is derived for the maximum power that a given generator $G_{i}$ can in inject into a system described by impedance network (includes all branches and loads) and $K$ voltage sources $\left(\bar{V}_{1}, \bar{V}_{2}, \ldots, \bar{V}_{K}\right)$ where each source is connected to a node of power injection and $K$ is the number of generators. The boundary of maximum injectable power for $G_{i}$ is determined by fixing both magnitude and angle of complex voltages $\bar{V}_{k}$ ( for $k=\{1, \ldots, K\} \backslash\{i\}$ ) at all other nodes of power injection and determine at which phase angle $\delta_{i}\left(\bar{V}_{i}=\left(V_{i} \angle \delta_{i}\right)\right)$ the point of maximum injectable power occurs for $G_{i}$. It turns out that the maximum power that a given generator $G_{i}$ can inject into a node of constant voltage magnitude can be expressed in terms of the generator's injection impedance $\bar{Z}_{i n j, i}$ and the system Thevenin impedance $\bar{Z}_{T h, i}$ seen from the node of injection (a node of constant steady state voltage magnitude). The boundary for generator $G_{i}$ maximum injectable power in steady state, expressed in terms of injection impedance, can be written as the following polar equation [10]:

$$
Z_{i n j, i}=-\frac{Z_{T h, i} \sin \theta}{\sin \phi_{T h, i}}
$$

where $\phi_{T h, i}$ is the angle of the system Thevenin impedance $\bar{Z}_{T h, i}$ and $\theta$ is the angle of the injection impedance $\bar{Z}_{i n j, i}$. It can be shown that (1) represents the operating conditions when the phase angle difference $\delta_{i}$ between the node of injection for $G_{i}$ and the Thevenin voltage behind $\bar{Z}_{T h, i}$ becomes equal $180-\phi_{T h, i}$.

If (1) is plotted for $\theta$ in the range $[0,2 \pi]$, the boundary will appear as an circle with radius $r=Z_{T h, i} /\left(2 \sin \phi_{T h, i}\right)$. The boundary circle intercepts the points where $\bar{Z}_{i n j, i}=-\bar{Z}_{T h, i}$ and $\bar{Z}_{i n j, i}=\bar{Z}_{T h, i}^{*}$, as well as the origin of the injection impedance plane. An operation point outside the circle indicates an stable condition where a relative increase in the phase angle at the node of injection results in increased power injection into the node. An operation point inside the circle, represents an unstable conditions characterized by a decrease in the injected power as the phase angle increases at the node of injection.

\section{B. Assessment Criteria and Outline of Assessment Approach}

By utilizing (1), the aperiodic small signal stability of a given generator $G_{i}$ can be described by the following set of inequalities [10]:

$$
\left|\frac{\bar{Z}_{i n j, i} \cdot\left(2 \sin \phi_{T h, i}\right)+j \cdot Z_{T h, i}}{Z_{T h, i}}\right| \begin{cases}>1 & \text { Stable operation } \\ =1 & \text { At the boundary } \\ <1 & \text { Unstable operation }\end{cases}
$$

The value of $\bar{Z}_{i n j, i}$ can be determined directly from PMUmeasurements taken at the terminal of the machine $G_{i}$. The value of $\bar{Z}_{T h, i}$ is dependent on load and branch impedance values in the grid and is determined by operations on the extended network admittance matrix [10]. The elements in the admittance matrix are updated for every PMU-snapshot received, resulting in an updated value of $\bar{Z}_{T h, i}$ for every new snapshot.

Utilizing the criteria in (2), an approach for assessing the aperiodic small-signal stability of the system generators can be outlined consisting of the steps shown as algorithm 1.

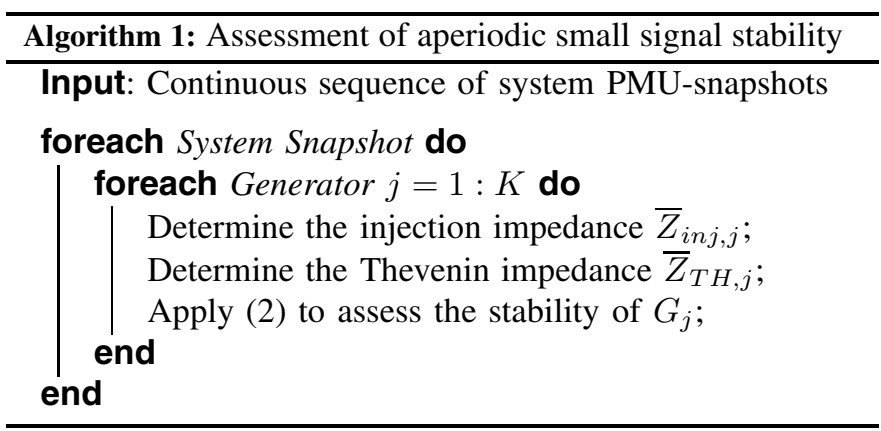




\section{Stability Margins as an Early Warning Indicator}

The presented assessment criteria in (2) enables a detection of when the stability boundary is crossed by a given generator. For the purpose of obtaining an early warning for an emerging stability problem, it is not sufficient to merely detect when the stability boundary is crossed. Instead, it is desirable to receive a warning in time before that actual boundaries are crossed, such that appropriate remedial control actions can be taken to avoid instability.

For the purpose of providing an early warning for an emerging stability problem, the margin from an observed operating point to its corresponding stability boundary may become a useful indicator. In the suggested assessment method, the stability boundary related to a given operating point $\left\{\bar{Z}_{i n j, i}, \bar{Z}_{T h, i}\right\}$, is represented in the injection impedance plane. A margin from the point $\bar{Z}_{i n j, i}$ to its critical boundaries expressed in term of injection impedance does not provide a useful physical interpretation of the distance to instability. Instead, it is more useful to derive margins that are expressed in quantities such as active power or as percentage power margin to the maximum.

The assessment method is based on that a given machine $G_{i}$ injects power into a node of constant steady state voltage magnitude $\bar{V}_{i}$ and the system is represented by a Thevenin voltage source $\bar{E}_{T h, i}$ behind the Thevenin impedance $\bar{Z}_{T h, i}$. In the following, the Thevenin voltage is used as phase angle reference and $\bar{V}_{i}=V_{i} \angle \delta_{i}$. As previously mentioned, the stability boundary given by (1) is represented by a line of constant voltage phase angle in the injection impedance plane where $\delta_{i}=180-\phi_{T h, i}$. By utilizing this, the margin from a observed operating point $\left\{\bar{Z}_{i n j, i}, \bar{Z}_{t h, i}\right\}$ to its corresponding boundaries can be expressed in terms of active power.

To do so, the active power injection is expressed as:

$$
P_{i n j, i}=\frac{E_{T h, i} V_{i}}{Z_{T h, i}} \cos \left(\delta_{i}+\phi_{T h, i}\right)-\frac{V_{i}^{2}}{Z_{T h, i}} \cos \left(\phi_{T h, i}\right)
$$

A power injection is represented by a negative value of $P_{i n j, i}$. As mentioned previously, the maximum injected power occurs when $\delta_{i}=180^{\circ}-\phi_{T h, i}$ and can therefore be expressed as:

$$
\hat{P}_{i n j, i}=-\frac{E_{T h, i} V_{i}}{Z_{T h, i}}-\frac{V_{i}^{2}}{Z_{T h, i}} \cos \left(\phi_{T h, i}\right)
$$

An active power margin $\Delta P_{i n j, i}$ becomes:

$\Delta P_{i n j, i}=\hat{P}_{i n j, i}-P_{i n j}=-\frac{E_{T h, i} V_{i}}{Z_{T h, i}}\left(\cos \left(\delta_{i}+\phi_{T h, i}\right)+1\right)$

Utilizing that $V_{i}$ and $\delta_{i}$ can be expressed as:

$$
\begin{aligned}
V_{i} & =E_{T h, i} \cdot\left|\frac{\bar{Z}_{i n j, i}}{\bar{Z}_{T h, i}+\bar{Z}_{i n j, i}}\right| \\
\delta_{i} & =\arg \left\{\frac{\bar{Z}_{i n j, i}}{\bar{Z}_{T h, i}+\bar{Z}_{i n j, i}}\right\}
\end{aligned}
$$

(5) can be expressed as a percentage margin to the maximum $\% \Delta P_{i n j, i}$ from merely knowing the values of the impedance pair $\left\{\bar{Z}_{i n j, i}, \bar{Z}_{t h, i}\right\}$. The percentage margin is expressed as:

$$
\begin{aligned}
\% \Delta P_{i n j, i} & =\frac{\Delta P_{i n j, i}}{\hat{P}_{i n j, i}} \cdot 100 \% \\
& =\frac{\cos \left(\delta_{i}+\phi_{T h, i}\right)+1}{1+\left|\frac{\bar{Z}_{i n j, i}}{\bar{Z}_{T h, i}+\bar{Z}_{i n j, i}}\right| \cos \left(\phi_{T h, i}\right)} \cdot 100 \%
\end{aligned}
$$

The above margin describe how much the active power injection can be increased as the phase angle $\delta_{i}$ (relative to the Thevenin voltage) is increased to its critical value at $\delta_{i}=180-\phi_{T h, i}$, while other system variables are fixed. The margin is therefore derived considering changes in only one system variable, the voltage phase angle $\delta_{i}$.

This is different from how stability margins are derived by means of continuation methods where the system is stressed in a particular direction by applying some pre-defined loading and dispatch patterns. The choice of stress patterns are usually based on operational experience, where the daily consumption patterns are usually the same and well known by the system operators.

Even though the above suggested stability margins, derived from the phase angle margin $\Delta \delta_{i}$, are not obtained by applying specific "normal" stress patterns they do anyhow provide a useful information. The method is intended to provide a stability assessment during critical or emergency operating conditions following a severe disturbance. During such conditions, it is not likely that the normal stress patterns would give the most likely stress direction of the system, since many other control and load restoration mechanisms have a more significant role in such situations. The actual stress direction might be dominated by the actions of ULTC-transformers and other devices that try to restore the pre-disturbance consumption. This means that the "normal" stress direction is not suitable for determining a margin to the system stability boundary in such situations. On the other hand, the element-wise margins (5)-(8) provide a valuable information concerning which of the system generators is operating close to, or approaching its stability boundaries. Such information could be used to take remedial actions to avoid an emerging instability.

\section{Visualization of multiple operating points}

The suggested method performs an element-wise assessment of the generators aperiodic small signal stability. In a system having $K$ generators, $K$ individual Thevenin impedances $\bar{Z}_{T h, i}(i=1, \ldots, K)$ are determined resulting in that $K$ different stability boundaries are used for the overall system assessment. Previously, it was mentioned that the stability boundaries are circular, which makes it possible to normalize all of the $K$ boundaries in such way that they appear as the unit circle centered at the origin of the normalized injection impedance plane. Doing so, all of the $K$ operating points for the generators can be visualized in the same screen shot where all of the points are held against the same stability boundary.

In [10] expression are derived for lines of constant $P, Q, V$ and $\delta$ in the injection impedance plane for a given Thevenin impedance $\bar{Z}_{T h, i}$. It would be desirable if the $K$ operating 
points could be normalized in such way, that some of the lines of constant $P, Q, V$ or $\delta$ are also represented in the normalized injection impedance plane. In that way, the characteristic lines would provide a useful information regarding the operating conditions of all of the $K$ normalized operating points and enhance the informative visualization of the system operating conditions.

In the following, it will be shown how a given operating point $\left\{\bar{Z}_{i n j, i}, \bar{Z}_{T h, i}\right\}$ can be mapped into a normalized injection impedance plane such that specific characteristic lines are preserved after the mapping. To do that, the mapping:

$$
f:\left\{\bar{Z}_{i n j, 0}, \bar{Z}_{t h, 0}\right\} \rightarrow\left\{\bar{Z}_{i n j, \star},\left(Z_{t h, 0} \angle 90^{\circ}\right)\right\}
$$

has to be determined where the following properties of the mapped operating point are preserved:

- The normalized voltage magnitude $V_{i} / E_{T h, i}$ is the same for both operating points

- The phase angle margin $\Delta \delta_{i}$ to the critical boundary is preserved after the mapping

In the subsequent expression, $\bar{Z}_{i n j, 0}$ and $\bar{Z}_{T h, 0}$ are used to denote original variables while $\bar{Z}_{i n j, \star}$ denotes the mapped variable. In [10], it is shown that the mapping in (9) of an arbitrary operating point that fulfills the criteria above can be expressed as:

$$
\bar{Z}_{i n j, \star}=\frac{1}{\frac{1}{\bar{Z}_{i n j, 0}}+\frac{1}{\bar{Z}_{T h, 0}}\left(1-e^{j\left(\phi_{T h, 0}-90^{\circ}\right)}\right)}
$$

The stability boundaries for aperiodic small signal stability appears as a circle with the following characteristics:

- With radius $r=Z_{T h, 0} /\left(2 \sin \phi_{T h, 0}\right)$

- The center of the circle is located on the imaginary axis where $X_{i n j, 0}=-r$

For enabling visualization of multiple operating points in the same injection impedance plane, the stability boundaries are normalized in such way that they appear as a unit circle centered at the origin of a normalized impedance plane. For that purpose, all impedance values $\bar{Z}_{i n j, \star}$ have to be shifted by $r$ in the direction of the imaginary axis and scaled down by factor $r$. For achieving that, the expression (10) can be manipulated into:

$$
\bar{Z}_{i n j, \star, p u}=\frac{\bar{Z}_{i n j, \star}}{r}+j=\frac{2 \bar{Z}_{i n j, \star}}{Z_{t h, 0}}+j
$$

By applying (11), the boundary and the characteristic lines for constant $\Delta \delta_{i}$ and $V_{i} / E_{T h, i}$ are the same for all of the $K$ mapped operating points $\left\{\bar{Z}_{i n j, i}, \bar{Z}_{T h, i}\right\}$ in the normalized injection impedance plane. Figure 3 shows few lines of constant $\Delta \delta_{i}$ and $V_{i} / E_{T h, i}$ that apply to all operating points that have been mapped by applying (10) and (11).

\section{Simulation And Test Results}

In order to test the presented assessment method, two different instability scenarios were simulated where in the first scenario a very simple system was considered while in the second scenario a slightly modified IEEE 14 bus system was used.

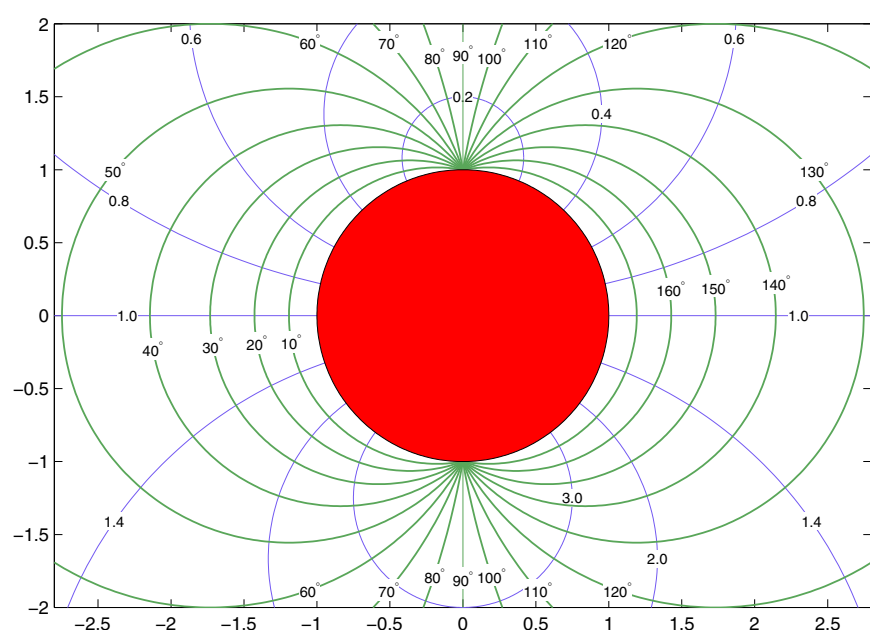

Fig. 3. The normalized injection impedance plane when (10) and (11) have been applied on an arbitrary operating point. The stability boundary always appears as an unit circle at the origin. Lines of constant voltage ratio $V_{i} / E_{T h, i}$ and lines of constant phase angle difference $\Delta \delta_{i}$ from the critical boundaries are valid for all operating points $\left\{\bar{Z}_{i n j, i}, \bar{Z}_{t h, i}\right\}$ that have been normalized by (10) and (11)

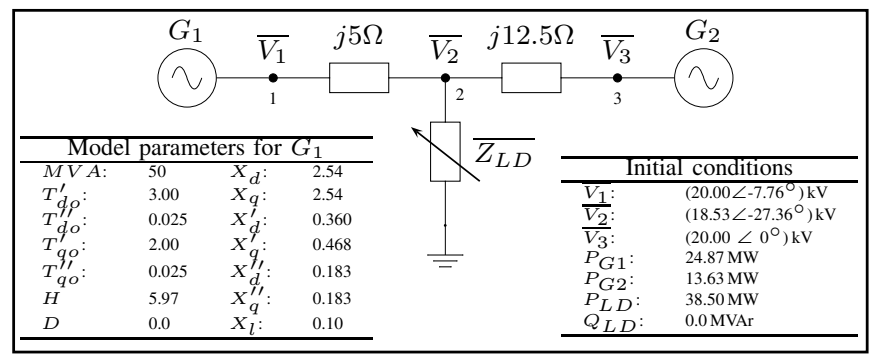

Fig. 4. Case A: The three-bus system, used to test the suggested assessment method. The system voltage is $20 \mathrm{kV}$ line-to-line and the generator $G_{3}$ represents an infinite bus $(H \rightarrow \infty)$. Generator $G_{1}$ is manually excited and is operated with constant mechanical power input.

\section{A. Case A: Test of a Simple System}

1) System Description: Figure 4 shows a three-bus network inspired by [11] used for a simple test of the method. It consists of three $20 \mathrm{kV}$ busses; one load bus (bus 2) adjacent to two generator busses (busses 1 and 3). Generator $G_{2}$ at bus 3, represents an infinite bus where the voltage at bus 3 is constant during the analysis. Generator $G_{1}$ is a $50 M V A$ round rotor machine that operates with constant mechanical input power and manual excitation. The parameters of dynamic model for $G_{1}$ are provided in figure 4 .

The initial conditions for the simulation are provided in the figure, where the load is purely resistive of $38.5 \mathrm{MW}$ and $G_{1}$ produces $24.87 \mathrm{MW}$. In the following, a loss of synchronous operation of $G_{1}$ is provoked by applying two minor disturbances in the form of increase in load demand on bus 2 .

2) Simulation and Test Results: The time domain simulation was carried out in PSS/E (version 30) where the initial conditions from figure 4 and two small step decreases in the load impedance at bus 2 were applied to provoke an aperiodic small signal instability. The results from the time domain simulation are shown in figure 5 where plot (i) shows the applied changes in the load impedance that eventually 
(i) Load Impedance on bus $2\left(Z_{L D}[\Omega]\right)$

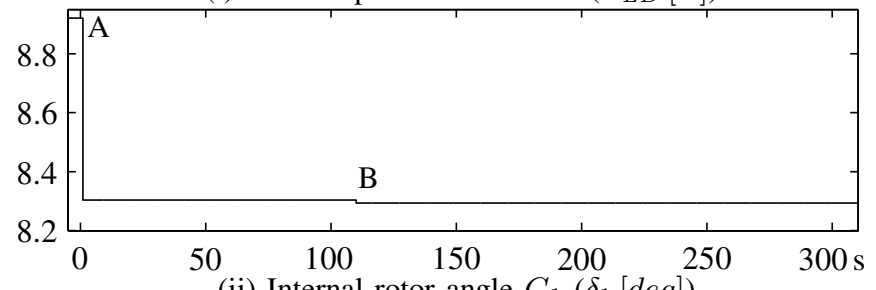

(ii) Internal rotor angle $G_{1}\left(\delta_{1}[\mathrm{deg}]\right)$

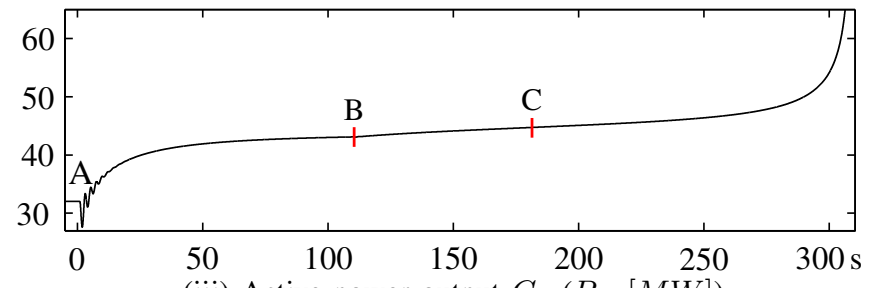

(iii) Active power output $G_{1}\left(P_{G 1}[M W]\right)$

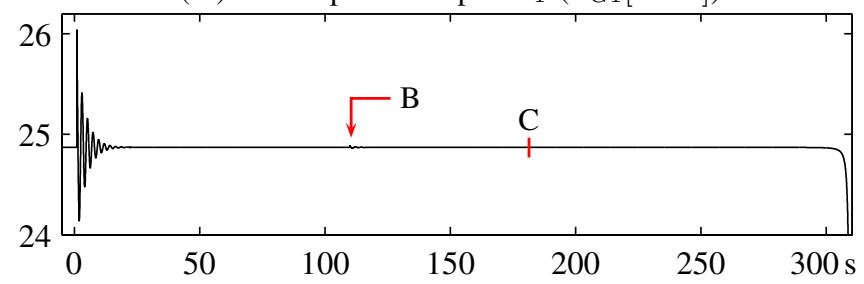

24.89 (iv) Active power output $G_{1}$, enlarged view $\left(P_{G 1}[M W]\right)$
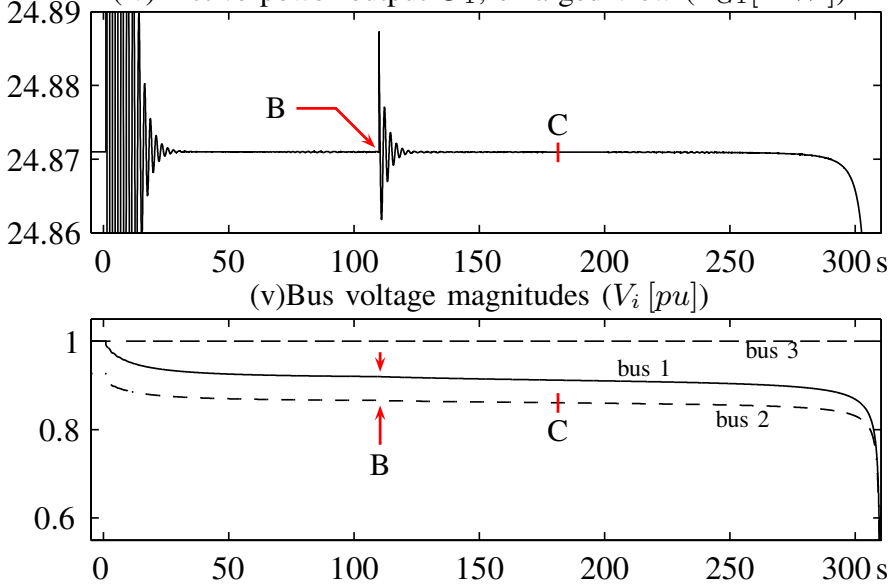

Fig. 5. Case A: The applied disturbances, the internal rotor angle deviation, the active power from $G_{1}$ and the bus voltage magnitudes, are shown from (i)-(iii) and (v), respectively. $A$ denotes the first disturbance, $B$ the second disturbance and $C$ the time of detected stability boundary crossover.

caused the system instability. The first disturbance was applied at $t=1 \mathrm{~s}$ (disturbance A) where the load impedance was changed from $8.9210 \Omega$ to $8.3039 \Omega$. The second disturbance was applied at the time $t=110 \mathrm{~s}$ (disturbance $\mathrm{B}$ ) where the impedance was changed from $8.3039 \Omega$ to $8.2936 \Omega$. It can be seen from the other plots that approximately $200 \mathrm{~s}$ passed from the second disturbance until a very sudden decrease in the system voltages occurred.

Disturbance $A$ brings machine $G_{1}$ very close to its stability boundaries, but the stability is preserved after the disturbance. It is the occurrence of the very small disturbance $B$, that causes eventually a loss of rotor angle stability.

The simulation results were used to test when the stability criteria in (2) detects a crossover of the stability boundary. The time domain simulation was carried out in such a way that values of line flows and bus voltages were stored in a output file for every $20 \mathrm{~ms}$ of the simulated time interval. The output data was used to generate synthetic PMU-measurements, which resulted in that a snapshot of the system conditions was obtained for every $20 \mathrm{~ms}$. For every simulated PMU-snapshot, the criteria in (2) was updated in order to determine when a boundary crossover occurs.

The time when the method detected instability is marked $C$ in the plots in figure 5 . The system boundary crossover occurs approximately $71 s$ after the second disturbance is applied. It is worth noticing that the time elapsed from the detection of the boundary crossover until the very sharp decline in voltage magnitude occurred is approximately $130 \mathrm{~s}$. The reason for this long time before the sharp decline in voltage magnitude is experienced is due to a very small imbalance between the applied mechanical torque and the induced electrical torque at the time of boundary crossover. This means that the machine angular acceleration is very small and very slowly increasing to begin with resulting in that approximately $130 \mathrm{~s}$ passed before the instability could be visually identified in the plots in figure 5 .

\section{B. Case B: Large Scale Test of IEEE 14 Bus System}

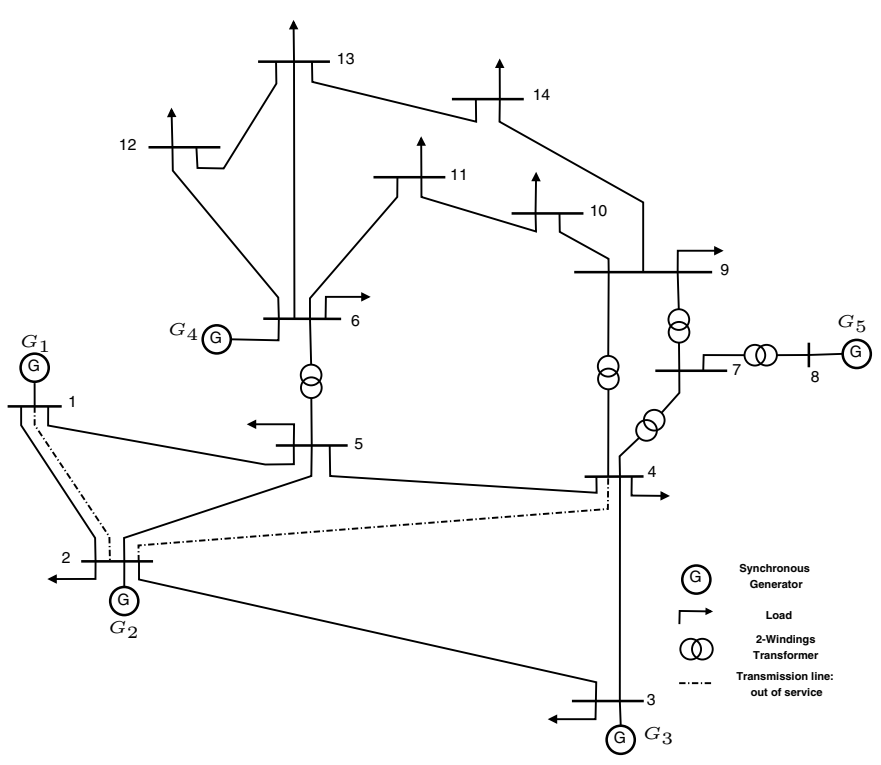

Fig. 6. Case B: One-line diagram of the modified IEEE 14 bus system.

1) System Description: The second system used for testing the assessment method is a slightly modified IEEE 14 bus system depicted in figure 6, where the initial configuration is shown. The system has been modified in such way, that the three synchronous condensers in the original system (on busses 3, 6 and 8) have been replaced by generators $G_{3^{-}}$ $G_{5}$. Generator $G_{5}$ is chosen to be very large and is used to represent a connection to a strong external grid. Consequently, to cope with the changes made to $G_{5}$, connections from bus 8 to busses 9 and 4 have been strengthen as well. The connection of bus 1 and 2 was originally represented by a single branch connection, which was replaced by two parallel branches in the modified test system. 


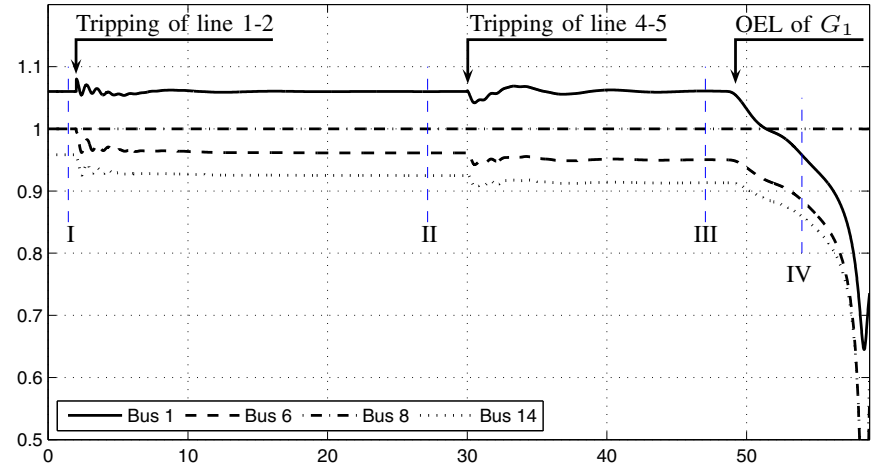

Fig. 7. Case B: Voltages at busses 1, 6, 8 and 14. Labels I-IV show the instance of time of the system snapshots analysed in figure8. The occurrence of the tripping of the two lines and the activation of the OEL of $G_{1}$ are marked in the figure.

In order to illustrate how the machines operating point are depending on the type of excitation control used, generators $G_{2}, G_{3}$ and $G_{4}$ are operated as manually excited machines, while $G_{1}$ and $G_{5}$ are operated with automatic voltage regulation and equipped with OEL for protection.

2) Simulation and Test Results: In order to provoke the occurrence of an aperiodic small signal stability, two disturbances were applied to the network:

- The branch 1-2 is tripped at $t=2 \mathrm{~s}$

- The branch 4-5 is tripped at $t=30 \mathrm{~s}$

Figure 7 depicts simulation results of the system voltages at busses 1, 6, 8 and 14. The second line trip resulted in that the excitation current of $G_{1}$ became higher than the threshold for the machine's OEL, causing that the OEL was activated at $t=48.5$. In seconds following, the system voltage magnitudes began to decline over a period of $10 s$ resulting in a rapid collapse in voltage magnitude.

Figure 8 shows the resulting stability assessment at four different snapshots I-IV as marked in figure 7. For each snapshot, the outlined procedure in algorithm 1 is followed. Equations 10-11 are then used to determine the normalized value of the machines injection impedance such that the stability boundary of all operating points appear as the unit circle.

Snapshot I shows the initial conditions where the actual operating point for machines $G_{2}-G_{4}$ is shown as small circular points while the actual operating points for $G_{1}$ and $G_{5}$ are outside viewable range of the plot. The squared point represents where the operating point of $G_{1}$ would be if its OEL would trigger and therefore this point is not the actual operating point of $G_{1}$. The percentage margin from the OEL point of $G_{1}$ to the critical stability margin is $6.8 \%$. The actual points of $G_{2}-G_{4}$ have a margin of $29.7 \%$ and higher.

Snapshot II, shows the situation after the first disturbance. All of the operating points have moved closer to the stability boundary. The point representing $G_{1}$, when its OEL has activated, is now $0.4 \%$. The positions of the initial operating points are shown in light gray color for indicating the relative movement of the operating points.
Snapshot III shows the situation after the second disturbance, but before the OEL has activated. It is worth noticing that the point for $G_{1}$, when the OEL has activated, has now crossed the stability boundary. This indicates that aperiodic instability of $G_{1}$ will be experienced if the machines OEL activates. The actual operating point of the machine $G_{1}$ appears now in the plot as a circular point having a $31.6 \%$ margin to the stability boundary.

Snapshot IV shows the situation when the OEL of $G_{1}$ has activated resulting in that the operating point inside the boundary circle becomes the actual operating point of $G_{1}$. Consequently, $G_{1}$ becomes unstable resulting in that generators $G_{1}$ together with $G_{2}-G_{4}$ separate from machine $G_{5}$ which represents a strong external system.

An early warning information for impending stability problems can be obtained from both snapshots II and III. Snapshot II provides the information that $G_{1}$ will be operating very close to the stability boundaries if its OEL activates or if there is switched from automatic to manual excitation of the machine. In this situation, it would be desirable to increase the safety margin for the operating point of concern. Snapshot III provides the information that the machine will become unstable if its OXL is activate and therefore remedial actions should be taken in order to avoid eventual instability.

\section{CONCLUSION}

A method for element-wise assessment of aperiodic small signal stability was presented in this paper. The method determines for each system generator, the maximum power that the generator can inject into the system in steady state. This boundary of maximum injectable steady state power is as well the generator's boundary of aperiodic small-signal rotorangle stability. If all power injections into the system network are represented at a node of constant voltage magnitude, the stability boundary for a given generator appears as a circle in the injection impedance plane. The circular shape of the boundary opens up for illustrative visualization of the operating conditions, where the conditions of all system generators is represented in the same screen shot. since a expression for the stability boundary was analytically derived, it was possible to derive as well an analytical expression of the generator's stability margin in terms of the the impedances $Z_{t h, i}$ and $Z_{i n j, i}$. The analytical expressions for the boundary and the stability margin enable a direct assessment of the generator conditions and hence, very short computational times.

Be representing the system conditions as an impedance network which is extended to nodes of constant voltage magnitude, enables that deterministic approaches can be used to determine $Z_{t h, i}$ and $Z_{i n j, i}$. Consequently, short computational times are required for the assessment process which makes the presented method suitable for real-time monitoring of the system generators operating points and their margin to the stability boundary.

The assessment method has the advantage that it can provide both an measure of the distance-to-instability and an information regarding which are the most critical machines in operation. The method provides therefore a strong basis for 

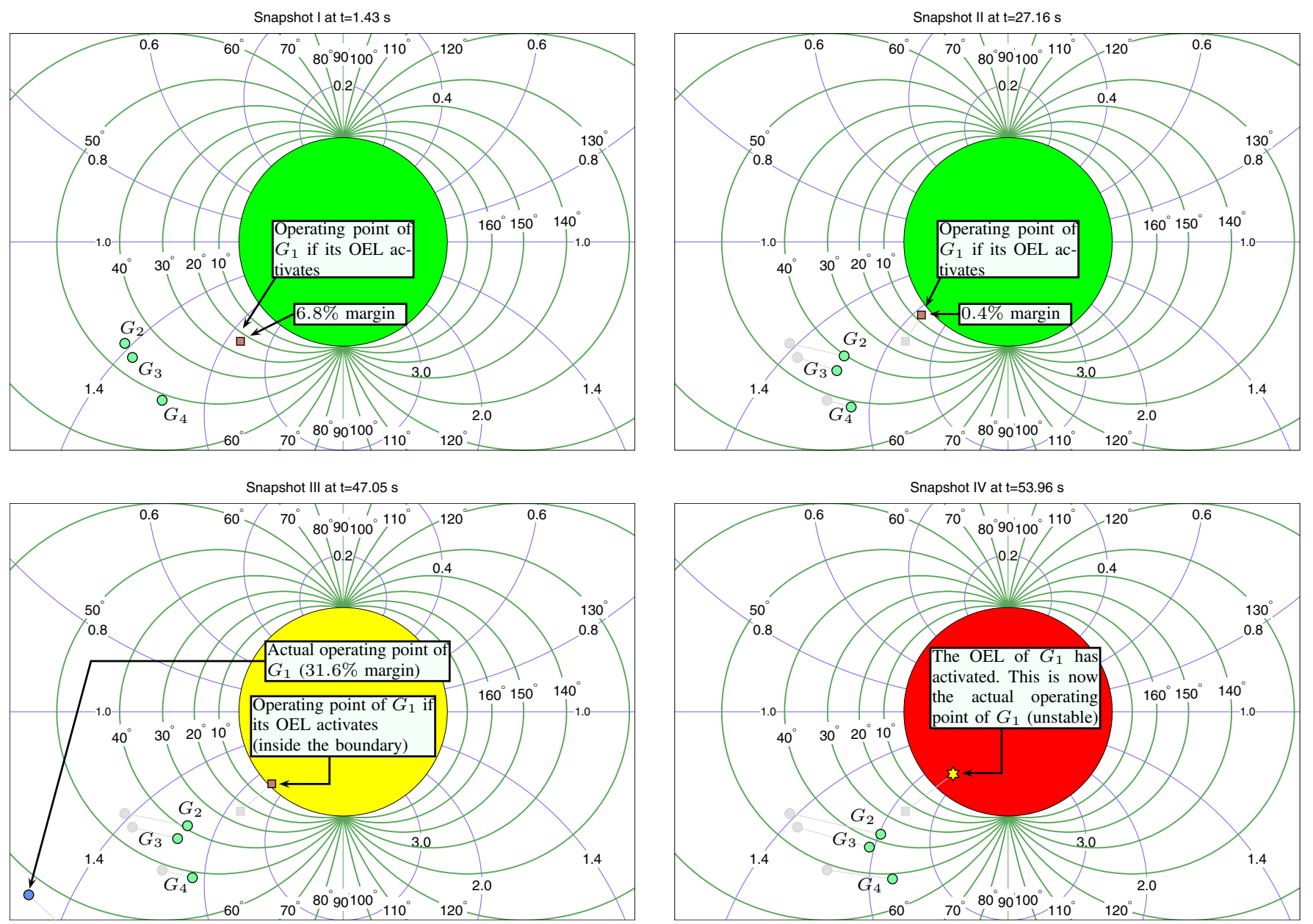

Fig. 8. Case B: Assessment of the system conditions at four different instances of time. The machines operating points are normalized according to (10)-(11) resulting in the same stability boundary to be applied for all points. The small circular points denote the actual operating point of a given machine, while the squared points represent the situation where a given machine would be if its OEL is activated.

the development of early warning system where the machines stability boundaries are monitored in real-time by the use of synchronized phasor measurements. Potential early warning indicators may be for example, when the observed margin to the stability boundary becomes smaller than a predefined minimum margin, or when the relative change in the stability margin over a given period of time is decreasing at a higher rate than some predefined maximum.

Furthermore, the assessment method opens up the possibility for development of methods for early prevention of instability. Since the element-wise assessment method provides an information of which system generators are critical, this information can be used for determining automatically preventive control actions that avoid the occurrence of system instability.

\section{REFERENCES}

[1] A. Phadke, J. Thorp, and M. Adamiak, "A New Measurement Technique for Tracking Voltage Phasors, Local System Frequency, and Rate of Change of Frequency," IEEE Transactions on Power Apparatus and Systems, vol. 102, no. 5, pp. 1025-1038, 1983.

[2] A. Phadke and J. Thorp, Synchronized Phasor Measurements and Their Applications. Springer Verlag, 2008.
[3] A. Phadke and R. de Moraes, "The Wide World of Wide-Area Measurement," IEEE Power and Energy Magazine, vol. 6, no. 5, pp. 52-65, 2008.

[4] S. Skok, I. Ivankovic, and Z. Cerina, "Applications Based on PMU Technology for Improved Power System Utilization," in Power Engineering Society General Meeting, 2007. IEEE, 2007, pp. 1-8.

[5] D. Novosel, V. Madani, B. Bhargava, K. Vu, and J. Cole, "Dawn of the grid synchronization," IEEE Power and Energy Magazine, vol. 6, no. 1, pp. 49-60, 2008.

[6] M. Glavic and T. Van Cutsem, "Wide-area detection of voltage instability from synchronized phasor measurements. part i: Principle," IEEE TRANSACTIONS ON POWER SYSTEMS, vol. 24, pp. 1408-1416, AUG 2009.

[7] — "Wide-area detection of voltage instability from synchronized phasor measurements. part ii: Simulation results," IEEE TRANSACTIONS ON POWER SYSTEMS, vol. 24, pp. 1417-1425, AUG 2009.

[8] K. Morison, L. Wang, and P. Kundur, "Power system security assessment," Power and Energy Magazine, IEEE, vol. 2, no. 5, pp. 30-39, 2004.

[9] Y. Makarov, S. Lu, X. Guo, J. Gronquist, P. Du, T. Nguyen, and J. Burns, "Wide Area Security Region Final Report," Pacific Northwest National Laboratory (PNNL), Richland, WA (US), Tech. Rep., 2010.

[10] H. Jóhannsson, "Development of early warning methods for electric power systems," Ph.D. dissertation, The Technical University of Denmark, Oct. 2010.

[11] C. Vournas, P. Sauer, and M. Pai, "Relationships between voltage and angle stability of power systems," Electrical power \& energy systems, vol. 18 , no. 8 , pp. $493-500,1996$. 